\title{
Survival and analysis of prognostic factors for hepatoblastoma: based on SEER database
}

\author{
Tie-Cheng Feng, Hong-Yan Zai, Wei Jiang, Qin Zhu, Bo Jiang, Lei Yao, Xin-Ying Li, Zhi-Ming Wang \\ Department of Liver and Thyroid Surgery, Xiangya Hospital, Central South University, Changsha 410008, China \\ Contributions: (I) Conception and design: T Feng; (II) Administrative support: None; (III) Provision of study materials or patients: None; (IV) \\ Collection and assembly of data: T Feng; (V) Data analysis and interpretation: T Feng; (VI) Manuscript writing: All authors; (VII) Final approval of \\ manuscript: All authors. \\ Correspondence to: Zhi-Ming Wang, MD; Xin-Ying Li, MD. Department of Liver and Thyroid Surgery, Xiangya Hospital, Central South University, \\ 87 Xiang Ya Road, Changsha 410008, China. Email: wzmxycsu@hotmail.com; lixinyingcn@126.com.
}

\begin{abstract}
Background: The goal of this study is to assess the newest survival of hepatoblastoma (HB) and the risk factors which impacted on survival by using the Surveillance, Epidemiology and End Results (SEER) database, also calculate the incidence of $\mathrm{HB}$ in recent years.

Methods: We calculate age-adjusted incidence of HB by using SEER 21 registries. Age, sex, race, tumor size, macrovascular involvement, multifocal tumor, distant metastasis, the way of treatment, and the survival were collected for survival and analysis of prognostic factors in SEER 18 registries. Survival curves, according to different factors, were obtained by Kaplan-Meier estimates. Multivariable Cox regression models were also built.

Results: The overall age-adjusted incidence of HB was 0.19 patients per 100,000 children with a statistically significant increase per year. Overall survival (OS) at 1-, 3- and 5-year for all patients were $89.3 \%, 84.6 \%$, and $81.9 \%$, respectively. Multivariate analysis showed tumor size $>5 \mathrm{~cm}$ [hazard ratio (HR), 8.271; 95\% confidence interval (CI), 1.134-60.310], multiple tumors (HR, 2.578; 95\% CI, 1.424-4.668) and no-surgery treatment (HR, 7.520; 95\% CI, 4.121-13.724) were independent indicators of poor prognosis. Only the age $\geq 2$-year-old (HR, 3.240; 95\% CI, 1.433-7.326) and multiple tumors (HR, 2.395; 95\% CI, 1.057-5.430) were the risk factors for the surgical treatment group.

Conclusions: The survival of patients with HB has been greatly improved in the recent years, and at the same time, due to the application of better chemotherapy, we should re-evaluate the traditional risk indicators of prognosis in order to better apply to the clinical.
\end{abstract}

Keywords: Hepatoblastoma (HB); Surveillance, Epidemiology and End Results (SEER); survival

Submitted Aug 23, 2019. Accepted for publication Sep 04, 2019.

doi: 10.21037/atm.2019.09.76

View this article at: http://dx.doi.org/10.21037/atm.2019.09.76

\section{Introduction}

Hepatoblastoma $(\mathrm{HB})$ is the most common primary liver cancer in children with rapid growth in recent years (1-3). Patients with HB are usually present at $0-4$ years and rare to see at $>15$ years (4). At present, the pathogenesis of HB is unclear, which may be related to very low body weight, tobacco intake, and some inherited syndromes like Beckwith-Wiedemann syndrome (5).
Complete tumor resection is the cornerstone of treatment for $\mathrm{HB}$, which offers the only chance for longterm survival. However, most of them were presented with an extensive unifocal or multifocal primary tumor or distant metastases at diagnosis, and lost the chance to surgery, leading to a very low 5 -year survival rate (20-30\%) $(6,7)$. In the past four decades, great changes have taken place in the treatment of HB. In the 1980s, cisplatin (PLA) and doxorubicin (DO) were introduced to treat 
HB and improved prognosis. After then, several groups made their efforts to establish the core of the treatment for HB: chemotherapy plus complete resection of the tumor. The International Childhood Liver Tumor Strategy Group (SIOPEL) designed pretreatment extent of disease (PRETEXT) radiographically stage and risk stratification to conduct clinical treatment for HB. Meantime, Children's Oncology Group (COG) also based on surgical findings to establish the stage for progress. Relying on these stages, it can help stratify the disease and facilitate the search for appropriate treatments. According to SIOPEL, the 5-year overall survival (OS) rate for $\mathrm{HB}$ can reach $75 \%$.

Although survival rates have improved dramatically, there are many problems to be solved. The effects of many pathological factors on survival are not well understood, such as age, tumor size, multiple tumors, macrovascular invasion, and distant metastases. Moreover, small samples do not adequately account for their true survival. The goal of this study is to assess the newest survival of $\mathrm{HB}$ and the risk factors which impacted on survival by using the Surveillance, Epidemiology and End Results (SEER) database, also calculate the incidence of $\mathrm{HB}$ in recent years.

\section{Methods}

The SEER database provides clinical information on many tumors in an effort to reduce the cancer burden (8-12). We chose SEER 21 Regs Limited-Field Research Data + Hurricane Katrina Impacted Louisiana Cases, Nov 2018 Sub (2000-2016) to calculate age-adjusted incidence, which was largest geographic coverage availableapproximately $36.7 \%$ of the US population (based on 2010 census). SEER*Stat software (version 8.3.5; National Cancer Institute, Bethesda, MD, USA) was used to analyze incidence rates and trends from 2004 to 2016. All incidence data were age-adjusted and normalized to the 2000 US Standard Population. The annual percentage change (APC) was calculated using the weighted least squares method (https://seer.cancer.gov/).

To investigate the survival of HB and the risk factors, we chose SEER 18 Regs Research Data + Hurricane Katrina Impacted Louisiana Cases, Nov 2018 Sub (1975-2016), because this database contained the most risk factors. SEER database contains high-quality follow-up and related clinical information. For accurate enough survival information, this research extracted patient data from 2004 to 2011, and the follow-up time was more than 5 years.
According to the International Classification of Diseases for Oncology, third edition (ICD-O-3), the code of $\mathrm{HB}$ was 8970 . The age range is under the age of 19 years. The information of sex, race (whites, blacks, others), the size of the tumor, macrovascular involvement, multifocal tumor, distant metastasis, the way of treatment, and the survival were collected. The methods of treatment were classified as no-surgery, resection, and liver transplantation (LT). We excluded cases that no information provided in the extent of the disease and the way of treatment.

Statistical analysis was carried out by using IBM SPSS Statistics 25.0 (IBM Corp., Armonk, NY, USA). The survival rate of $\mathrm{HB}$ was calculated by using the KaplanMeier method, and the survival curve was drawn. The logrank test was used to formally test the differences. Cox's regression method was used for the multifactor analysis. Significant standard is $\mathrm{P}<0.05$.

\section{Results}

\section{The age-adjusted incidence of $\mathrm{HB}$}

In SEER 21 registries, 757 patients were identified from 2004 to 2016. The overall age-adjusted incidence of HB was 0.19 patients per 100,000 children with a statistically significant increase per year. The APC was $2.53 \%$ [95\% confidence interval (CI), 1.15-3.93\%; $\mathrm{P}<0.05$ ] (Figure 1).

\section{Patient demographics}

According to the selection criteria, a total of 302 cases were included in this study. Male patients accounted for $65.6 \%$, more than women. Most patients were under the age of 2 years, and over the age of 2 years accounted for only $36.1 \%$. The most common race is white, accounting for $79.5 \%$. Resection is still the most common treatment method, accounting for about $66.9 \%$. The detailed information is shown in Table 1.

\section{Survival and univariate analysis for all patients}

OS at 1-, 3- and 5-year for all patients were $89.3 \%, 84.6 \%$, and $81.9 \%$, respectively. There was no significant difference in sex $(\mathrm{P}=0.492)$. In the beginning, the survival curve of age was grouped for six: 0-, 1-, 2-, 3-, 4-, and >4-year. However, there was no significant difference in this classification. However, by observing the survival curve, we found the 


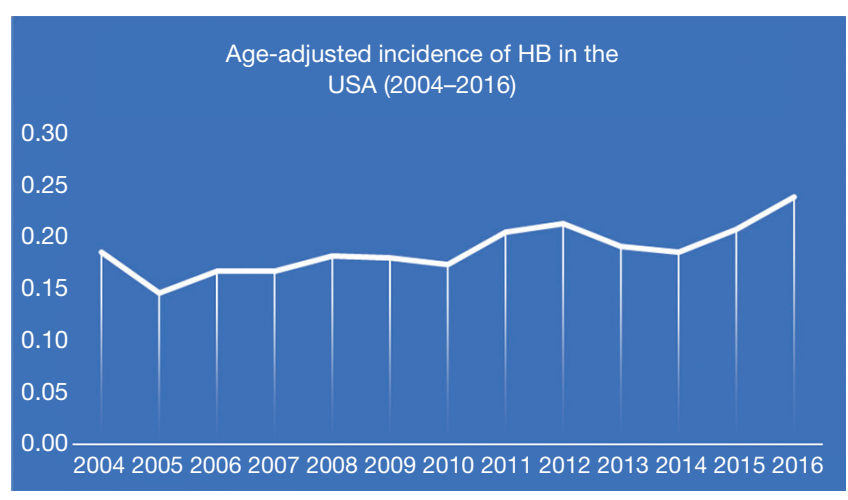

Figure 1 Age-adjusted incidence of HB in the USA (2004-2016). The incidence of $\mathrm{HB}$ has increased over time (APC, 2.53\%; $\mathrm{P}<0.05)$. HB, hepatoblastoma; APC, annual percentage change.

survival of children under the age of 1-year and 1-year was close and better to the other four groups. So, we redraw the curve of age by two groups: $<2$ - and $\geq 2$-year-old, and the difference were statistically significant $(\mathrm{P}=0.009)$. Resection compared with LT had a similar prognosis, and statistics proved this. The survival of the no-surgery group was the worst, 1-, 3-, 5-year survival rate was 53.2\%, 40.4\%, 35.8\%, respectively; 1-, 3-, 5-year survival of the resection group and LT was $96.0 \%, 92.9 \%, 89.8 \%$ and $96.2 \%, 92.5 \%$, $92.5 \%$, respectively. A single tumor had better survival than multiple tumors, and distant metastases had worse survival than no distant metastases. The tumor size of $\leq 5 \mathrm{~cm} \mathrm{had}$ very excellent survival, and the 5 -year OS was $97.1 \%$. There was no statistical significance in the macrovascular involvement and no macrovascular involvement group $(\mathrm{P}=0.966)$. The detailed information is shown in Table 2 . The survival curves are shown in Figures 2-10.

\section{Multivariate analysis for all patients}

Multivariate analysis showed tumor size $>5 \mathrm{~cm}$ [hazard ratio (HR), 8.271; 95\% CI, 1.134-60.310], multiple tumors (HR, 2.578 ; 95\% CI, 1.424-4.668) and no-surgery treatment (HR, 7.520; 95\% CI, 4.121-13.724) were independent indicators of poor prognosis. Age, sex, distant metastases, and macrovascular involvement were not prognostic indicators. The detailed information is shown in Table 3.

\section{Univariate and multivariate analysis for the surgical treatment group}

In order to further identify the prognostic factors of the
Table 1 Basic characteristics of patients from the SEER database

\begin{tabular}{|c|c|}
\hline Characteristics & Frequency, n (\%) \\
\hline \multicolumn{2}{|l|}{ Sex } \\
\hline Female & $104(34.4)$ \\
\hline Male & $198(65.6)$ \\
\hline \multicolumn{2}{|c|}{ Age at diagnosis } \\
\hline$<2$-year-old & $193(63.9)$ \\
\hline$\geq 2$-year-old & $109(36.1)$ \\
\hline \multicolumn{2}{|l|}{ Race } \\
\hline White & $240(79.5)$ \\
\hline Black & $19(6.3)$ \\
\hline Others & $43(14.2)$ \\
\hline \multicolumn{2}{|c|}{ Distant metastases } \\
\hline No & $221(73.2)$ \\
\hline Yes & $81(26.8)$ \\
\hline \multicolumn{2}{|l|}{ Tumor size } \\
\hline$\leq 5 \mathrm{~cm}$ & $36(11.9)$ \\
\hline$>5 \mathrm{~cm}$ & $233(77.2)$ \\
\hline Unknown & $33(10.9)$ \\
\hline \multicolumn{2}{|c|}{ Multiple or single } \\
\hline Single & $182(60.3)$ \\
\hline Multiple & $64(21.2)$ \\
\hline Unknown & $56(18.5)$ \\
\hline \multicolumn{2}{|c|}{ Macrovascular invasion } \\
\hline Yes & $33(10.9)$ \\
\hline No & $244(80.8)$ \\
\hline Unknown & $25(8.3)$ \\
\hline \multicolumn{2}{|l|}{ Surgery type } \\
\hline No-surgery & $47(15.6)$ \\
\hline Resection & 202 (66.9) \\
\hline LT & $53(17.5)$ \\
\hline
\end{tabular}

SEER, Surveillance, Epidemiology and End Results; LT, liver transplantation.

surgical treatment group, the resection group and the transplantation group were analyzed. Univariate analysis showed that the age $\geq 2$-year-old, distant metastases, multiple tumors, and tumor size $>5 \mathrm{~cm}$ were the risk factors of prognosis. However, in multivariate analysis, only the age 
Page 4 of 12

Feng et al. Survival and analysis of HB

Table 2 Survival and univariate analyses of prognostic factors of $\mathrm{HB}$

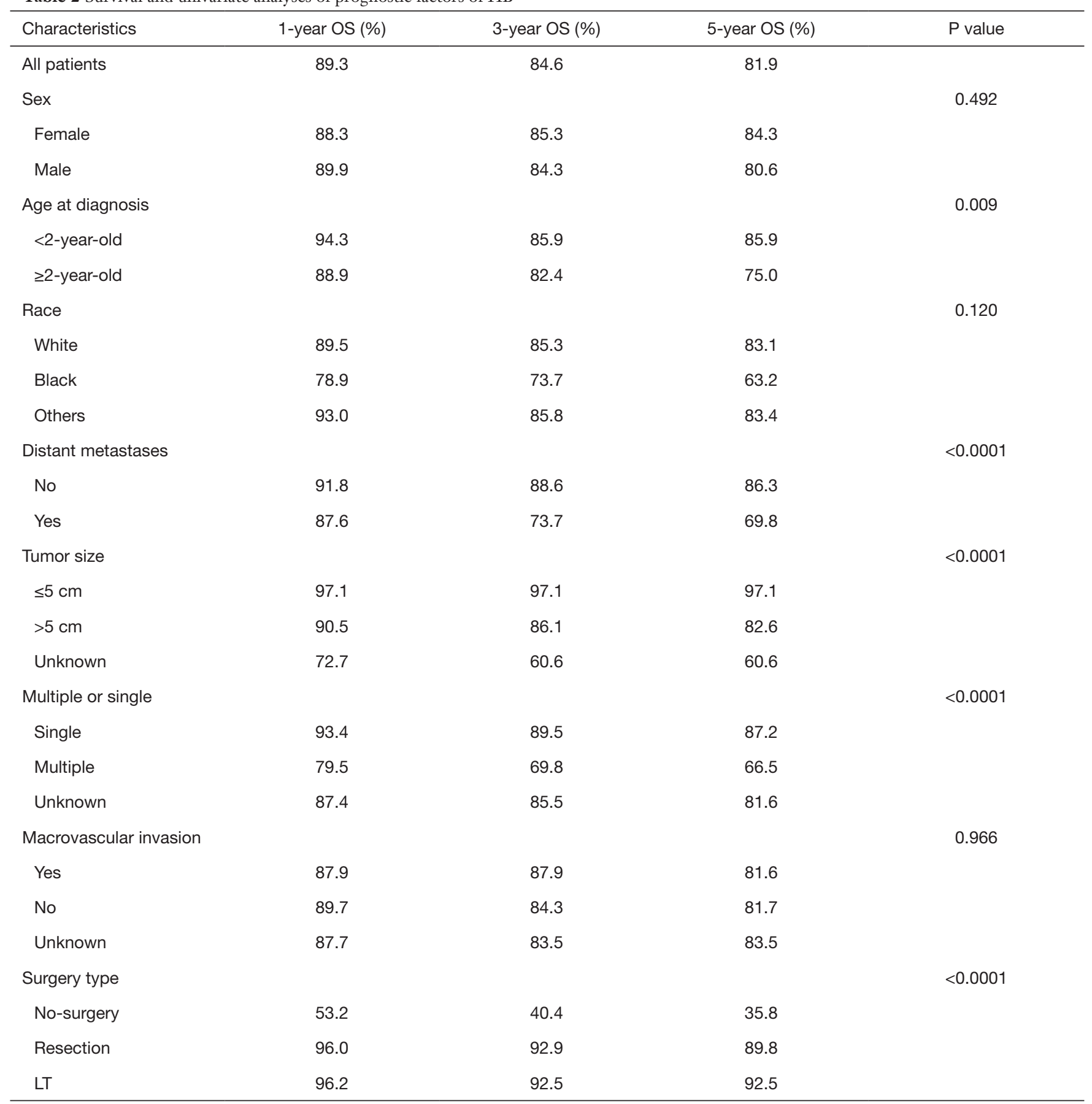

HB, hepatoblastoma; OS, overall survival; LT, liver transplantation. 


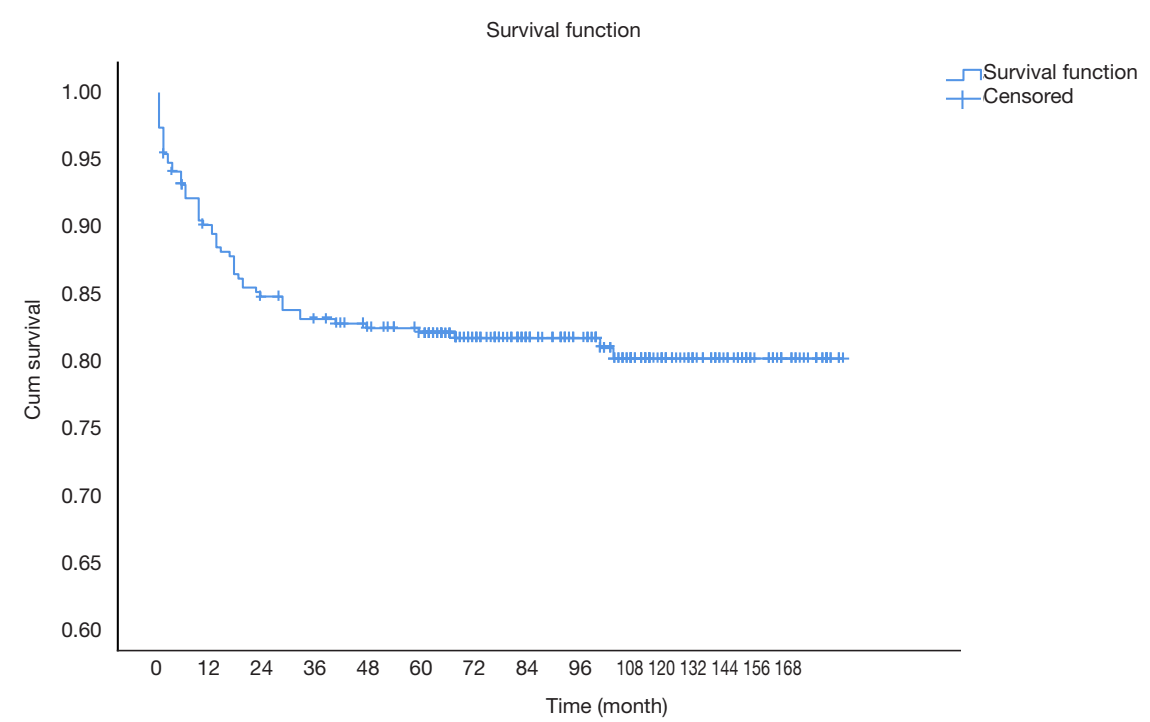

Figure 2 Survival curves for all HB. The 1-, 3- and 5-year OS of all patients was 89.3\%, 84.6\% and 81.9\%, respectively. HB, hepatoblastoma; OS, overall survival.

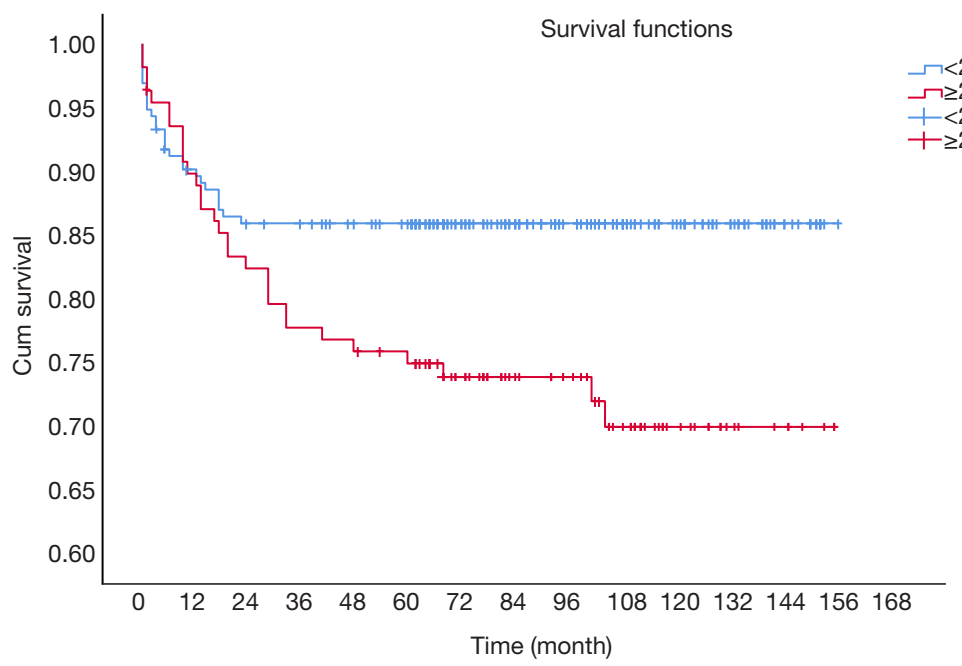

Figure 3 Survival curves for HB which were stratified according to age. $<2-v s$. $\geq 2$-year-old, $\mathrm{P}=0.009$. HB, hepatoblastoma.

22-year-old (HR, 3.240; 95\% CI, 1.433-7.326) and multiple tumors (HR, 2.395; 95\% CI, 1.057-5.430) were the risk factors for the surgical treatment group. The detailed information is shown in Tables 4,5.

\section{Discussion}

Although HB is the most common type of primary liver malignancy in children, its incidence is still very low. According to our results, the overall age-adjusted incidence of $\mathrm{HB}$ was 0.19 patients per 100,000 children. However, it is worth noting that its incidence is still increasing year by year, although we cannot find out the exact cause of this.

The prognosis of HB was gloomy 40 years ago. Because of multiple lesions or distant metastases at the time of diagnosis, radical surgery cannot be performed. Children usually died within six months after diagnosis, and the OS rate is less than $30 \%$. The change in treatment is mainly based on a series of studies conducted by SIOPEL. In 2000, neoadjuvant therapy was used to reduce the tumor 


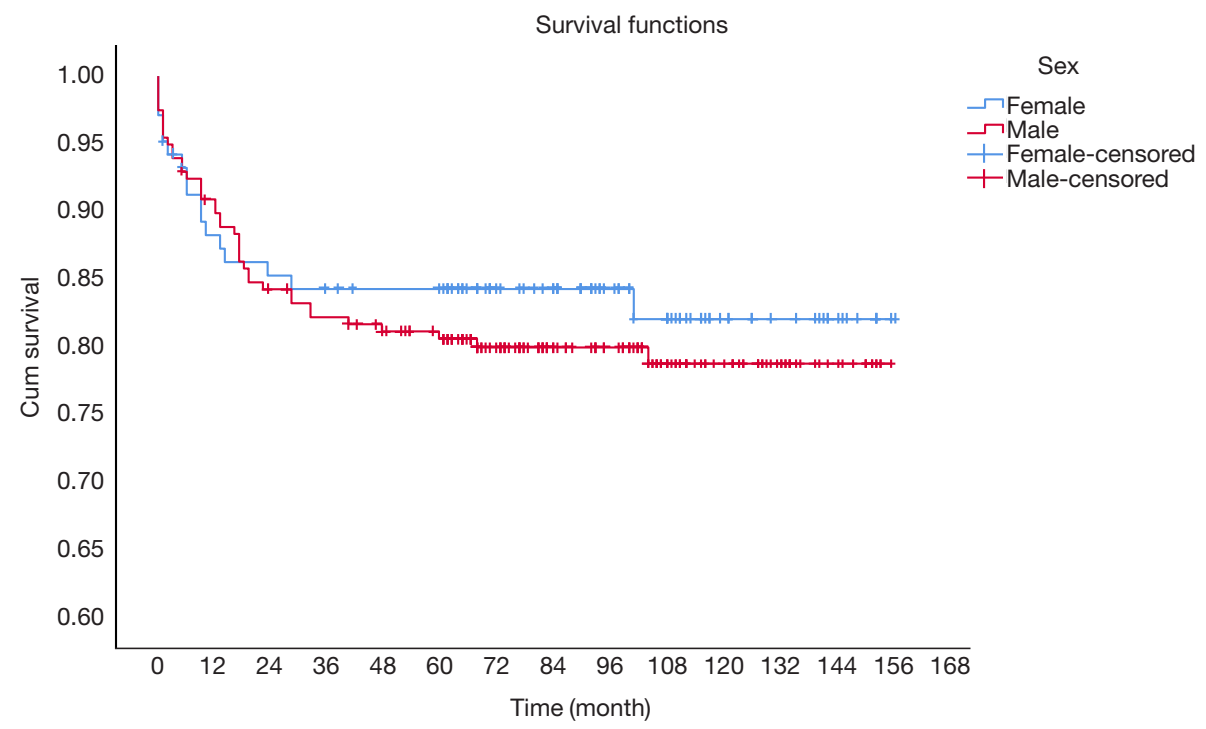

Figure 4 Survival curves for $\mathrm{HB}$ which were stratified according to sex. Female vs. male, $\mathrm{P}=0.492$. HB, hepatoblastoma.

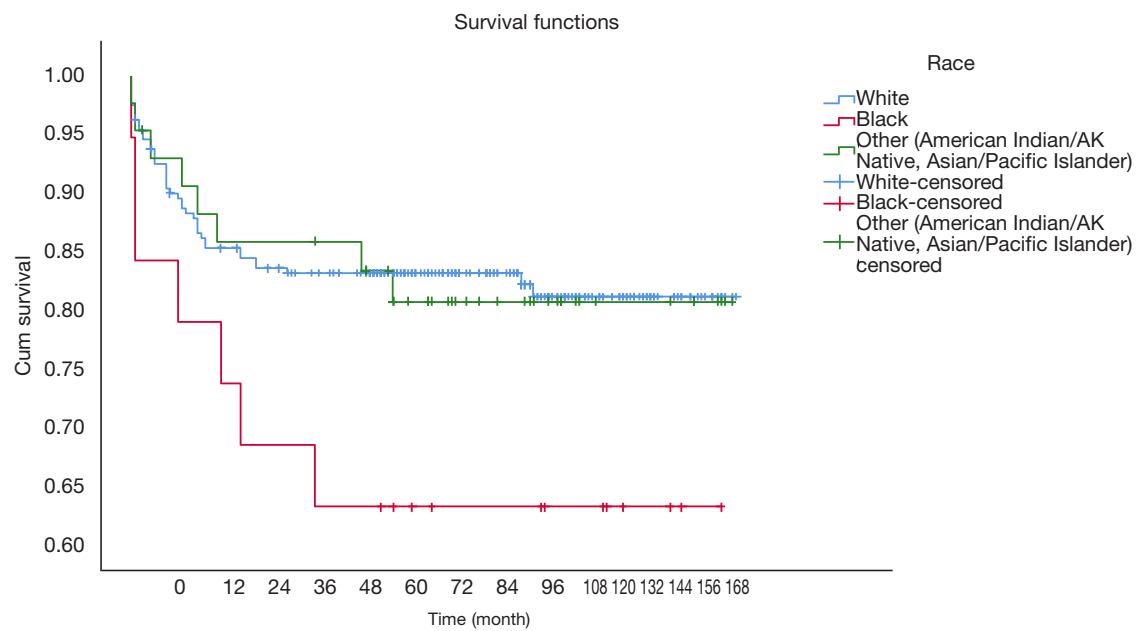

Figure 5 Survival curves for $\mathrm{HB}$ which were stratified according to the race. White $v s$. black, $\mathrm{P}=0.041$; white $v s$. other race, $\mathrm{P}=0.906$; black $v s$. other race, $\mathrm{P}=0.120$. HB, hepatoblastoma.

stage before surgery, and the 5 -year OS was $75 \%$. Since then, the status of neoadjuvant chemotherapy in the treatment of HB has been established, and survival has begun to increase. Previous studies based on the SEER database showed that the overall 5 -year survival of $\mathrm{HB}$ was $52-63 \%(1,13,14)$. Our study shows that the 5 -year OS of all patients was $81.9 \%$, significantly higher than that of previous studies. The reason for this phenomenon may lie in the use of surgical techniques and medical devices and better chemotherapy regimens. By studying HB at different periods, Horton et al. (14) also found that the 5 -year OS in $1973-1982$ was only $36 \%$, while the OS reached $63 \%$ in 1983-2005. This is similar to our standpoint.

No-surgery has become the most important factor affecting survival. The 5 -year OS of the resection group can reach $89.8 \%$, while only $35.8 \%$ in no-surgery group. The prognosis of the resection group was similar to that of the transplant group, which was similar to previous studies (15). Age has become an important prognostic indicator in many cancer studies. Although age was not 


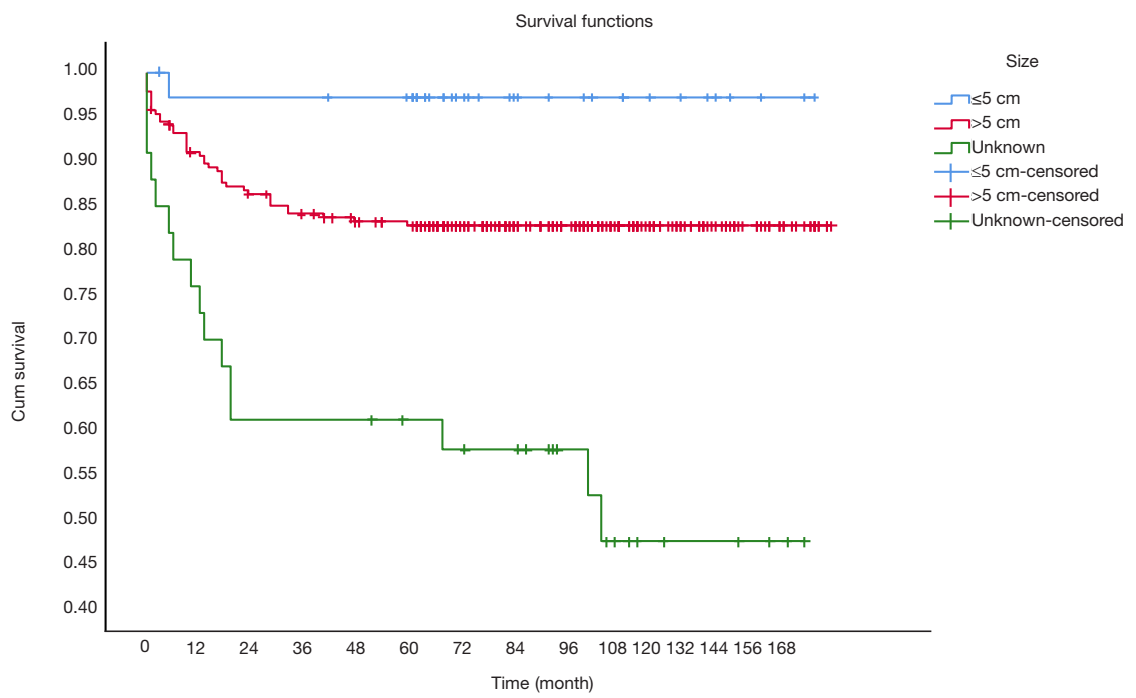

Figure 6 Survival curves for $\mathrm{HB}$ which were stratified according to tumor size. $\leq 5 v s .>5 \mathrm{~cm}, \mathrm{P}=0.031$. HB, hepatoblastoma.

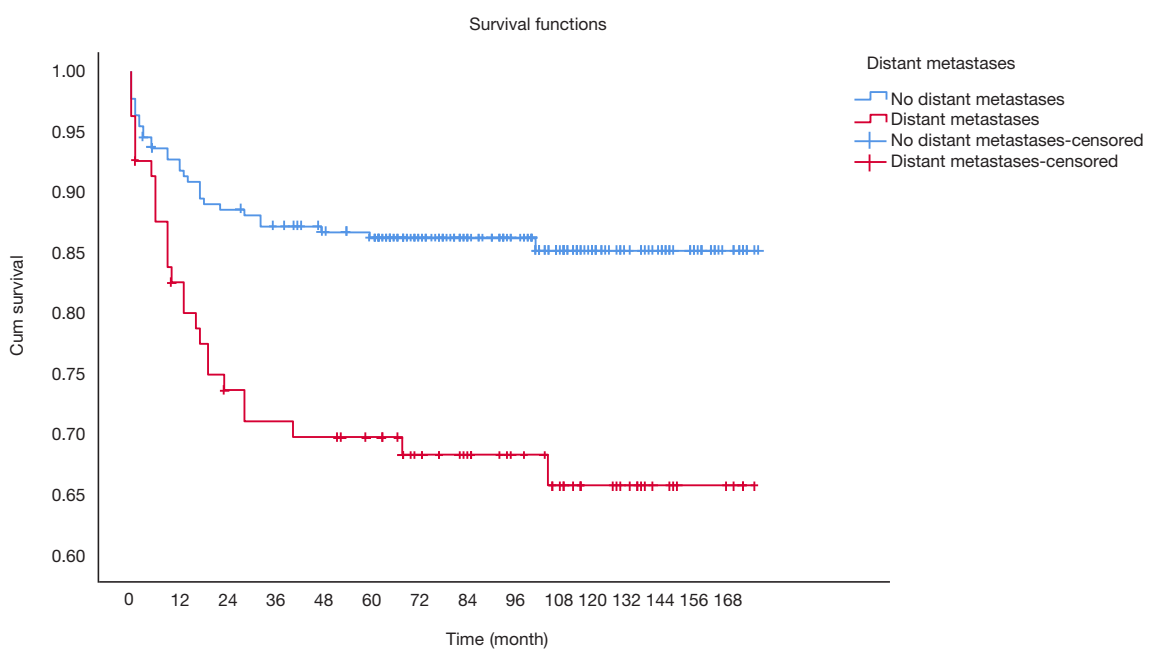

Figure 7 Survival curves for HB which were stratified according to distant metastases. No distant metastases vs. distant metastases, $\mathrm{P}<0.0001$. HB, hepatoblastoma.

considered as a risk factor in the initial PRETEXT stage, age became an important stratification factor in the recent stages by Children's Hepatic tumors International Collaboration (CHIC), and cut-off values were 3 - and 8 -year (16). We draw the survival curves by age group and found that the survival of 1 - and $<1$-year group were better than those older than 2-year, suggested that 2-year may be the cut-off value that affects the prognosis of $\mathrm{HB}$. Although age was not an independent predictor in the multivariate analysis for all patients, $\geq 2$-year-old was the only risk indicators in the multivariate analysis of the surgical treatment group.

In previous studies, distant metastasis, and macrovascular involvement were important prognostic factors. In our study, macrovascular involvement was not a risk factor either in univariate analysis or multivariate analysis. At the same time, distant metastasis of the tumor has prognostic significance only in univariate analysis. There were two possible reasons for these results. One may be the data were too few to distinguish variable (the macrovascular 


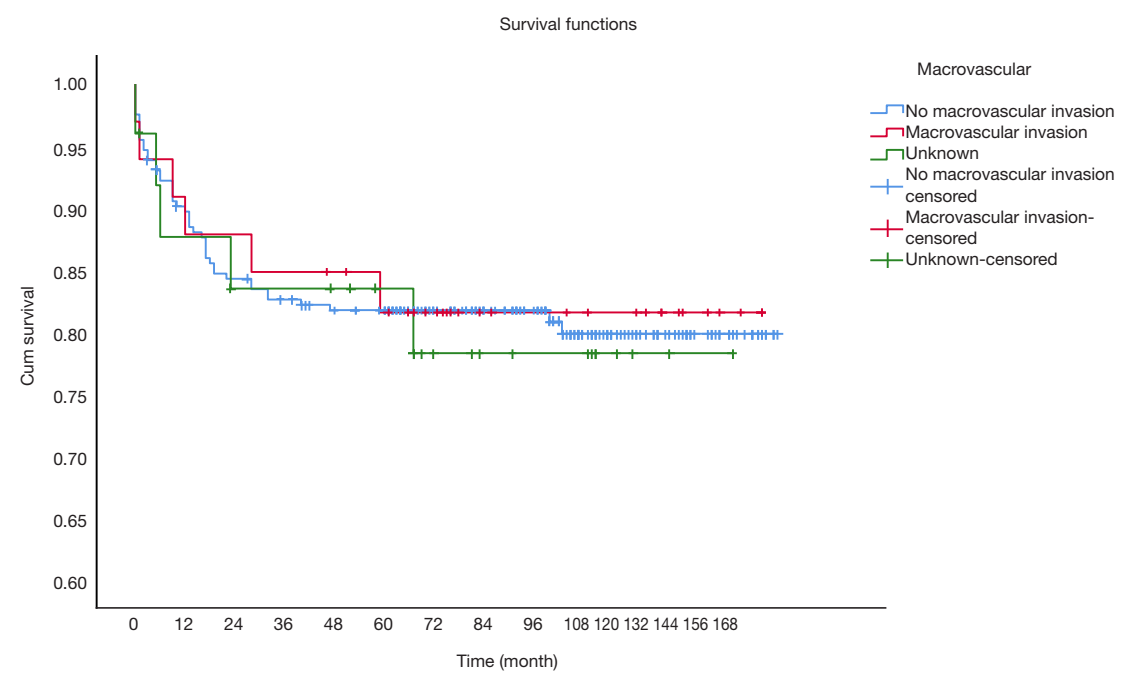

Figure 8 Survival curves for HB which were stratified according to the macrovascular invasion. No macrovascular invasion $v s$. macrovascular invasion, $\mathrm{P}=0.887$. HB, hepatoblastoma.

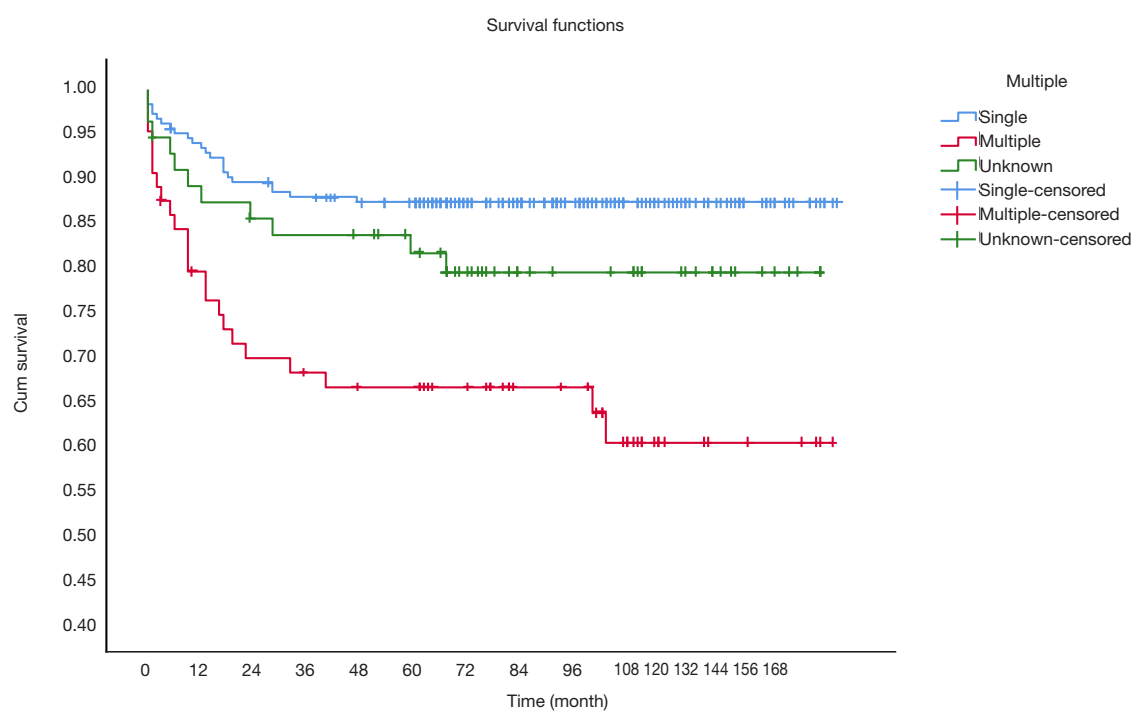

Figure 9 Survival curves for $\mathrm{HB}$ which were stratified according to multiple tumors or not. Single tumor vs. multiple tumors, $\mathrm{P}<0.0001$. HB, hepatoblastoma.

involvement group had 33 cases). The other reason may be that the impact of these factors was less important by using chemotherapy. More and more researchers believed that tumor response to chemotherapy was an important marker for predicting long term survival $(17,18)$. In the latest stratified PRETEXT staging, PRETEXT IV patients with no distant metastases, <3-year and alpha-fetoprotein (AFP) $>100 \mathrm{ng} / \mathrm{mL}$, with or without traditional risk factors (such as macrovascular invasion, multifocal, extrahepatic invasion and other factors) all had similar prognosis (16).

Similarly, the current view is that even if there is distant metastasis at the time of diagnosis, if the tumor is sensitive to chemotherapeutic drugs, surgical resection or transplantation can still be used to achieve good survival. Our study also confirmed this (the survival of the distant metastasis group was $85.2 \%$ after surgery). We believe 


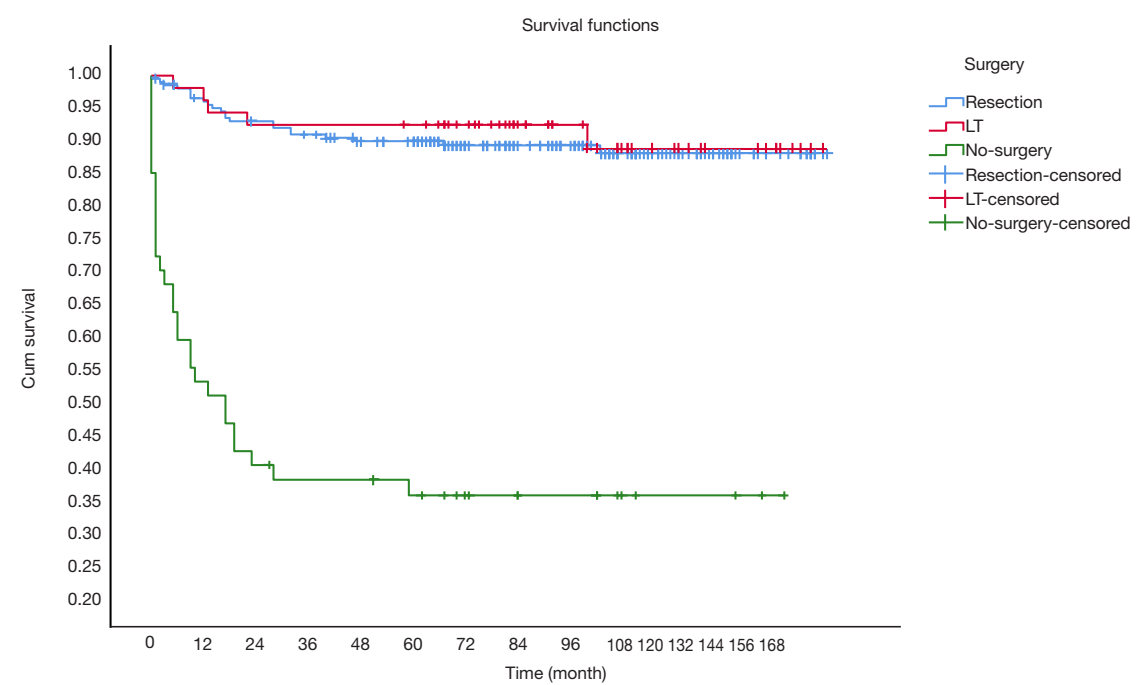

Figure 10 Survival curves for $\mathrm{HB}$ which were stratified according to treatment. Resection $v$ s. no-surgery, $\mathrm{P}<0.001$; resection $v$ s. LT, $\mathrm{P}=0.7$; LT vs. no-surgery, $\mathrm{P}<0.001$. HB, hepatoblastoma; LT, liver transplantation.

Table 3 Multivariate analyses of prognostic factors of $\mathrm{HB}$

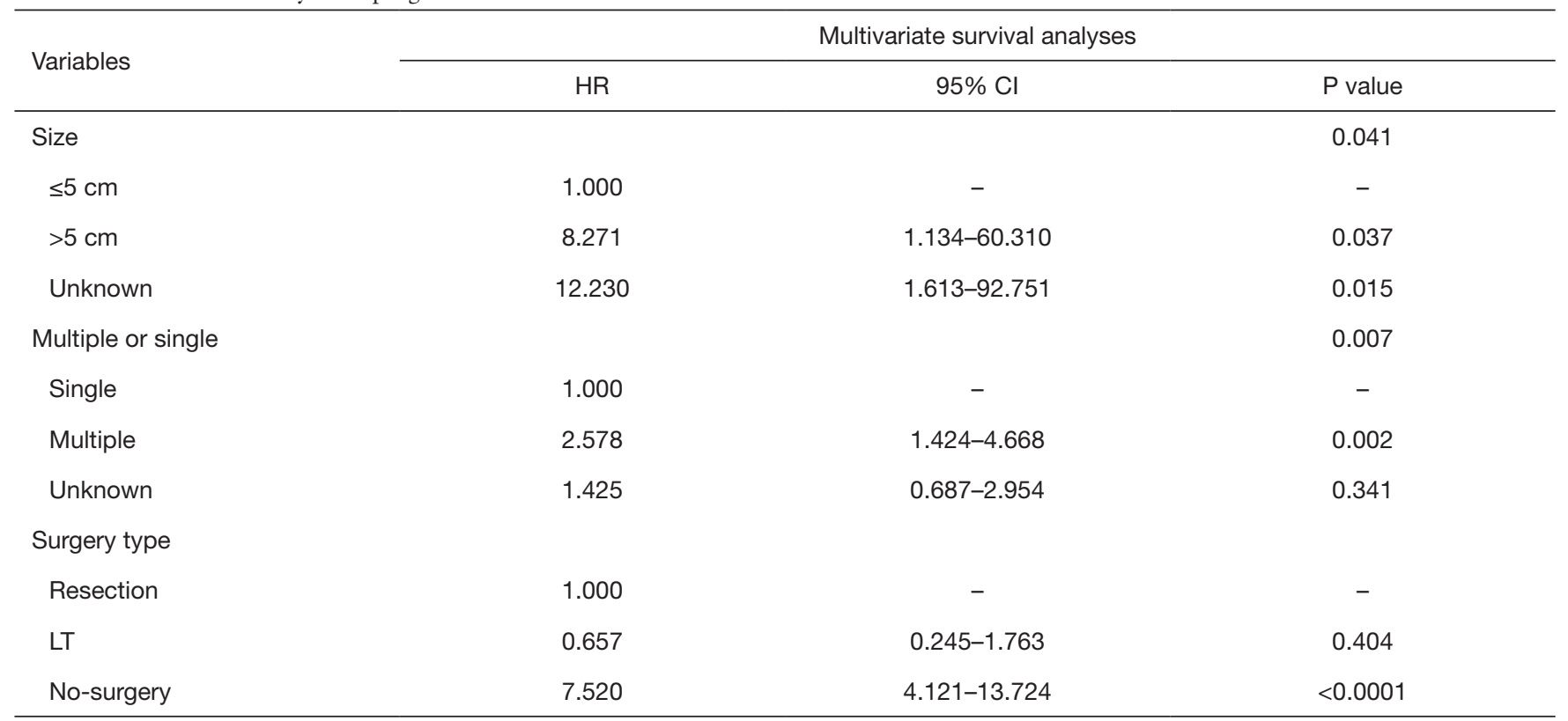

$\mathrm{HB}$, hepatoblastoma; $\mathrm{HR}$, hazard ratio; $\mathrm{Cl}$, confidence interval; $\mathrm{LT}$, liver transplantation.

that it is necessary to re-examine traditional risk factors, including distant metastasis, and more studies are needed to confirm our hypothesis.

Our research also has many shortages. First, because the SEER database does not provide internationally popular PRETEXT stages, there is no way to compare survival conditions with other clinical studies that adopt this staging approach. Second, we lack some clinical data, such as AFP, lymph node metastasis of the tumor, spontaneous rupture, and chemotherapy regimen. Third, due to retrospective studies, there is selective bias. In the end, in our article, there is some unknown data in some variables, such as 
Table 4 Survival and univariate analyses of prognostic factors of HB after surgery

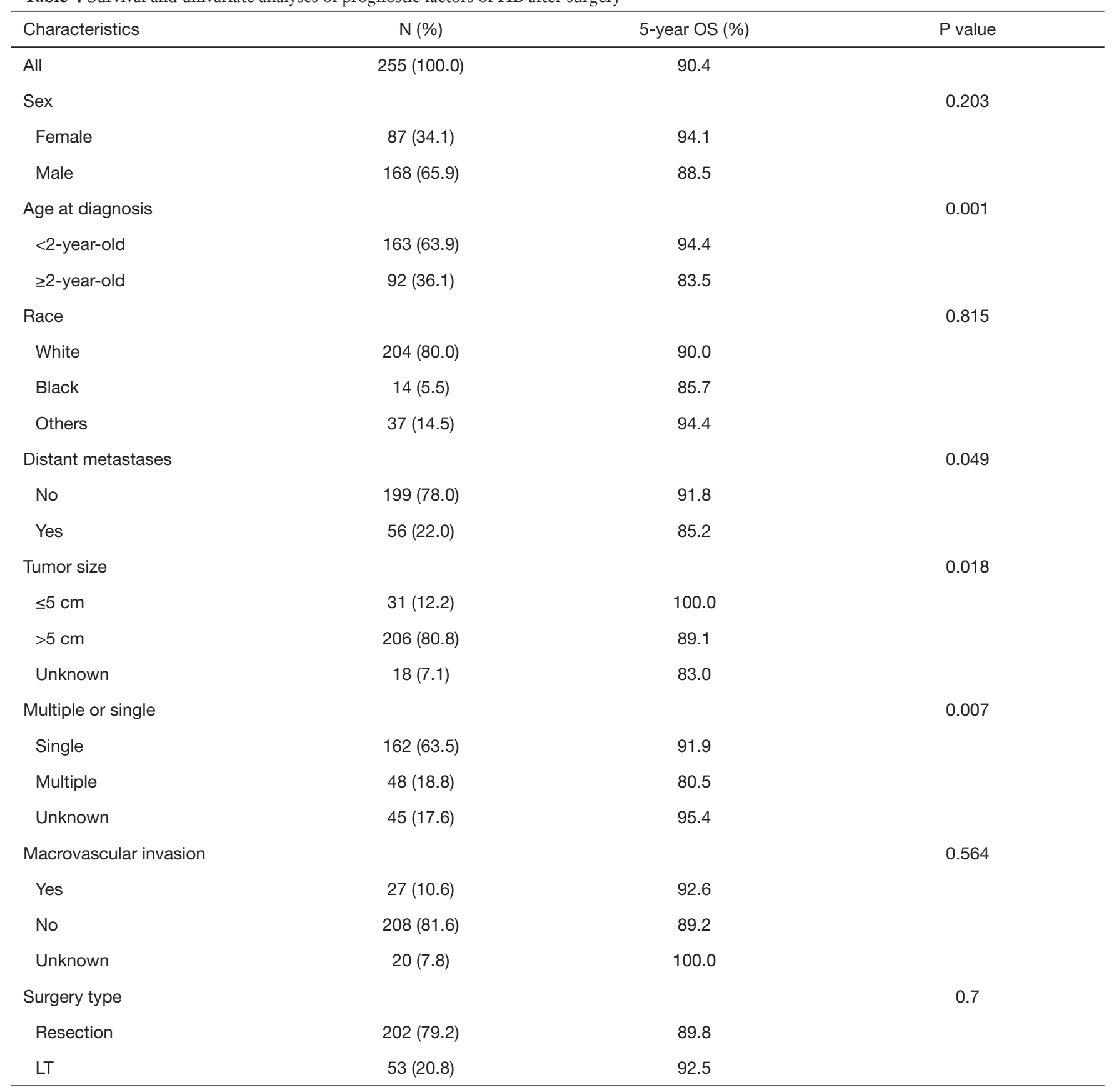

HB, hepatoblastoma; LT, liver transplantation.

tumor size, multiple tumors, and macrovascular involvement, which may have an impact on the results. However, even though the SEER database provides reliable follow-up, we believe these survival data are real and reliable.

In conclusion, through the above analysis, we believe that the survival of patients with HB has been greatly improved in the recent years, and at the same time, due to the application of better chemotherapy, we should reevaluate the traditional risk indicators of prognosis in order to better apply to the clinical. 
Table 5 Multivariate analyses of prognostic factors of HB after surgery

\begin{tabular}{lccc}
\hline Variables & \multicolumn{2}{c}{ Multivariate survival analyses } \\
\cline { 2 - 4 } & HR & $95 \% \mathrm{Cl}$ & $\mathrm{P}$ value \\
\hline Age & & - & 0.005 \\
$<2$-year-old & 1.000 & $1.433-7.326$ & - \\
$\geq 2$-year-old & 3.240 & - & - \\
Multiple or single & & -047 \\
Single & 1.000 & $1.057-5.430$ & 0.036 \\
Multiple & 2.395 & $0.190-2.367$ & 0.534 \\
Unknown & 0.670 & & - \\
\hline
\end{tabular}

$\mathrm{HB}$, hepatoblastoma; $\mathrm{HR}$, hazard ratio; $\mathrm{Cl}$, confidence interval.

\section{Acknowledgments}

None.

\section{Footnote}

Conflicts of Interest: The authors have no conflicts of interest to declare.

Ethical statement: The authors are accountable for all aspects of the work in ensuring that questions related to the accuracy or integrity of any part of the work are appropriately investigated and resolved.

\section{References}

1. Allan BJ, Parikh PP, Diaz S, et al. Predictors of survival and incidence of hepatoblastoma in the paediatric population. HPB (Oxford) 2013;15:741-6.

2. Linabery AM, Ross JA. Trends in childhood cancer incidence in the U.S. (1992-2004). Cancer 2008; 112:416-32.

3. Xie L, Onysko J, Morrison H. Childhood cancer incidence in Canada: demographic and geographic variation of temporal trends (1992-2010). Health Promot Chronic Dis Prev Can 2018;38:79-115.

4. Hung GY, Lin LY, Yu TY, et al. Hepatoblastoma incidence in Taiwan: a population-based study. J Chin Med Assoc 2018;81:541-7.

5. Spector LG, Birch J. The epidemiology of hepatoblastoma. Pediatr Blood Cancer 2012;59:776-9.

6. Exelby PR, Filler RM, Grosfeld JL. Liver tumors in children in the particular reference to hepatoblastoma and hepatocellular carcinoma: American Academy of Pediatrics Surgical Section Survey--1974. J Pediatr Surg 1975;10:329-37.

7. Hermann RE, Lonsdale D. Chemotherapy, radiotherapy, and hepatic lobectomy for hepatoblastoma in an infant: report of a survival. Surgery 1970;68:383-8.

8. Li Y, Zhao L, Güngör C, et al. The main contributor to the upswing of survival in locally advanced colorectal cancer: an analysis of the SEER database. Therap Adv Gastroenterol 2019;12:1756284819862154.

9. Feng SS, Li HB, Fan F, et al. Clinical characteristics and disease-specific prognostic nomogram for primary gliosarcoma: a SEER population-based analysis. Sci Rep 2019;9:10744.

10. Xiong Y, Cao H, Zhang Y, et al. Nomogram-predicted survival of breast cancer brain metastasis: a SEER-based population study. World Neurosurg 2019. [Epub ahead of print].

11. Xiao Q, Xiao H, Ouyang S, et al. Primary small cell carcinoma of the esophagus: Comparison between a Chinese cohort and surveillance, epidemiology, and end results (SEER) data. Cancer Med 2019;8:1074-85.

12. Pan $\mathrm{Y}, \mathrm{Lu} \mathrm{L}$, Chen J, et al. Analysis of prognostic factors for survival in patients with primary spinal chordoma using the SEER Registry from 1973 to 2014. J Orthop Surg Res 2018;13:76.

13. Darbari A, Sabin KM, Shapiro CN, et al. Epidemiology of primary hepatic malignancies in U.S. children. Hepatology 2003;38:560-6.

14. Horton JD, Lee S, Brown SR, et al. Survival trends in children with hepatoblastoma. Pediatr Surg Int 2009;25:407-12. 
15. McAteer JP, Goldin AB, Healey PJ, et al. Surgical treatment of primary liver tumors in children: outcomes analysis of resection and transplantation in the SEER database. Pediatr Transplant 2013;17:744-50.

16. Meyers RL, Maibach R, Hiyama E, et al. Risk-stratified staging in paediatric hepatoblastoma: a unified analysis from the Children's Hepatic tumors International
Collaboration. Lancet Oncol 2017;18:122-31.

17. Pimpalwar AP, Sharif K, Ramani P, et al. Strategy for hepatoblastoma management: Transplant versus nontransplant surgery. J Pediatr Surg 2002;37:240-5.

18. Browne M, Sher D, Grant D, et al. Survival after liver transplantation for hepatoblastoma: a 2-center experience. J Pediatr Surg 2008;43:1973-81.

Cite this article as: Feng TC, Zai HY, Jiang W, Zhu Q, Jiang B, Yao L, Li XY, Wang ZM. Survival and analysis of prognostic factors for hepatoblastoma: based on SEER database. Ann Transl Med 2019;7(20):555. doi: 10.21037/atm.2019.09.76 